\title{
Tinea unguium onychomycosis caused by dermatophytes: a ten-year (2005-2014) retrospective study in a tertiary hospital in Singapore
}

\author{
Shiu Ming Pang ${ }^{1,2,3}$, MBBS, FRCP, Jonathan Yi Yu Pang ${ }^{4}$, MB BCh BAO, MRCP, Stephanie Fook-Chong ${ }^{5}$, MSc, CStat,
} Ai Ling $\underline{T a n}^{6}$, MBBS, FRCPath

\begin{abstract}
INTRODUCTION Tinea unguium is a common nail infection. We conducted a retrospective ten-year study of the patient demographics and species distribution of dermatophytes causing tinea unguium in a tertiary hospital from Singapore. METHODS Results of fungal nail cultures were retrieved from our hospital's microbiology department. Samples from nail scrapings and clippings were inoculated onto agar plates (Sabouraud dextrose agar with chloramphenicol and Mycosel agar). Nail specimens that grew dermatophytes were included in the study.

RESULTS Overall, 229 (male: $n=164,71.6 \%$; female: $n=65,28.4 \%$ ) nail specimens grew dermatophytes. Mean patient age was 58 (range 18-93) years. A majority of specimens came from patients aged over 50 years $(n=162,70.7 \%)$ and 60-79 years $(n=100,43.7 \%)$. Ethnically, 160 (69.9\%) patients were Chinese, $36(15.7 \%)$ Indian, $18(7.9 \%)$ Malay and $15(6.6 \%)$ of other ethnicities. Among dermatophytes isolated were Trichophyton rubrum $(n=93,40.6 \%)$, Trichophyton mentagrophytes $(n=60,26.2 \%)$, unidentified Trichophyton spp. $(n=57,24.9 \%)$, Trichophyton tonsurans $(n=10,4.4 \%)$, Epidermophyton floccosum $(n=5,2.2 \%)$, Trichophyton verrucosum $(n=2,0.9 \%)$, Trichophyton soudanense $(n=1,0.4 \%)$ and Trichophyton violaceum $(n=1,0.4 \%)$.

CONCLUSION A majority of isolates were from elderly patients. Compared to Singapore's general population, patients of Indian and other ethnicities were over-represented for tinea unguium when compared to Chinese and Malay patients. Trichophyton rubrum was the most common dermatophyte isolated, while Trichophyton verrucosum, Trichophyton violaceum and Trichophyton soudanense were rare causes of tinea unguium.
\end{abstract}

Keywords: fungus, nail, onychomycosis, tinea unguium

\section{INTRODUCTION}

Nail conditions are a common complaint in general medical practice and dermatology consultations. Many patients see their family doctor or are referred to the dermatologist because they suspect their nail problem to be a fungal infection. A literature review by Ramesh et al concluded that onychomycosis, also known as tinea unguium, is a common fungal infection that constitutes $15 \%$ of all nail disturbances, while fungal infection of the nails constitutes $10 \%$ of all patients with dermatophytosis. ${ }^{(1)}$ In 2012, Hay and Baran estimated that only $18 \%-40 \%$ of all nail disorders were onychomycoses, and $30 \%$ of all dermatomycoses were nail infections. ${ }^{(2-4)}$ Even psoriasis of the nails can produce subungual hyperkeratosis, onycholysis and total nail dystrophy, ${ }^{(2)}$ which may mimic tinea unguium. Hence, as not all nail conditions are caused by fungal infection, it is important to confirm the diagnosis of onychomycosis before starting treatment.

English proposed that 'tinea unguium' should be confined to nail infections caused by dermatophytes, while 'onychomycosis' should include nail infections attributable to any fungus that may be dermatophyte, mould or yeast. ${ }^{(5)}$ Fungi cause onychomycosis by invading the nail plate or nail fold. There are a limited number of fungi capable of invading healthy nail keratin; these include some dermatophytes that possess keratinases, which allow them to use keratin as a substrate. Dermatophytes usually invade healthy nails. They may also invade dystrophic nails, which are especially common in elderly patients. ${ }^{(6)}$

The gold standard for diagnosing tinea unguium is to take a sample from the diseased nail and send it to a laboratory for culture test and microscopic examination. As the appearance of mycelium under the microscope alone may not indicate a dermatophyte as the causative agent, a final diagnosis can be established only after culture results become available. ${ }^{(6)}$ Any dermatophyte that is cultured from the nail is considered a pathogen and requires treatment.

In Singapore, Lim et al conducted a prospective study in 1992 on dermatophyte and non-dermatophyte onychomycoses at National Skin Centre, ${ }^{(7)}$ which provides outpatient care. The present retrospective study on patients with tinea unguium at Singapore General Hospital (SGH), Singapore, is the first to come close to that of Lim et al, studying similar patients in a hospital setting. The objective of our study was to determine the demographics and species distribution of dermatophytes among patients with tinea unguium in a tertiary hospital in an Asian, multiethnic country.

${ }^{1}$ Department of Dermatology, ${ }^{2}$ Transplant Centre, Singapore General Hospital, ${ }^{3}$ Duke-NUS Medical School, Singapore, ${ }^{4}$ Intense Care Unit, Nottingham Hospital, United Kingdom, ${ }^{5} \mathrm{Health}$ Services Research Unit, Division of Medicine, ${ }^{6}$ Department of Microbiology, Singapore General Hospital, Singapore

Correspondence: Dr Shiu Ming Pang, Senior Consultant, Department of Dermatology, Singapore General Hospital, 20 College Road, Academia, Singapore 169856. pang.shiu.ming@singhealth.com.sg 


\section{METHODS}

We conducted a retrospective study of patients with tinea unguium at SGH over a ten-year period between 1 January 2005 and 31 December 2014. SGH is a tertiary hospital situated within the SingHealth complex, which also houses National Cancer Centre Singapore, National Heart Centre Singapore, National Dental Centre Singapore, Singapore National Eye Centre, Outram Polyclinic (SingHealth) and Duke-NUS Medical School.

Culture results of nail specimens collected during the study period were retrieved from the Department of Microbiology, $\mathrm{SGH}$. The results of all dermatophyte nail infections were analysed. The source of the specimen was not stated as a fingernail or toenail in most of the requests, nor was it possible to differentiate between the two during our analysis. As the data for this retrospective study was retrieved from the microbiology department, the clinical features of tinea unguium infections were not available. Besides patient demographics, the species distribution of dermatophytes that caused tinea unguium in our patients was studied. Mycological cultures were used to confirm onychomycosis caused by dermatophytes.

Scrapings or cuttings from nails and nail beds were collected in sterile bottles and sent to the laboratory. The scrapings were picked up using sterile sticks and inoculated onto the agar plate. If the piece was too large, the nail would be cut into smaller pieces before being inoculated onto the agar plate (Sabouraud dextrose agar with chloramphenicol) and Mycosel agar containing cycloheximide and chloramphenicol. The agar plates were then incubated at $30^{\circ} \mathrm{C}$. The culture plates were examined once a week and discarded after four weeks if there was no growth. Any fungi that grew would be planted onto potato dextrose agar for gross and microscopic examinations. If necessary, urea hydrolysis was done. Trichophyton agar 1 and 4 were used to identify Trichophyton spp., the usefulness of which was limited to certain types of Trichophyton spp. only.

There are not many biochemical (phenotypic) tests that can differentiate Trichophyton spp. at the species level. Trichophyton was diagnosed mainly based on the gross morphology of the mould colonies (e.g. size, colour, texture and rate of growth) and their microscopic features. Trichophyton has macroconidia and microconidia arranged along the hyphae, which are used to identify the Trichophyton spp. Some Trichophyton spp. had poor sporulation on potato dextrose agar. When the macroconidia were lacking or not well formed and there were low numbers of microconidia despite being incubated for up to three weeks, the species name could not be identified and the specimen would be reported as unidentified Trichophyton spp. Trichophyton mentagrophytes was not subspeciated further, as it was difficult to subspeciate based on data from only the microscopic examination and phenotypic tests. These were the resources used for mould identification. Molecular sequencing would have been needed to subspeciate, but this was not routinely done for our nail specimens. At our centre, molecular sequencing is only done for deep-site specimens from fluids and blood culture, or when it is needed to guide management.

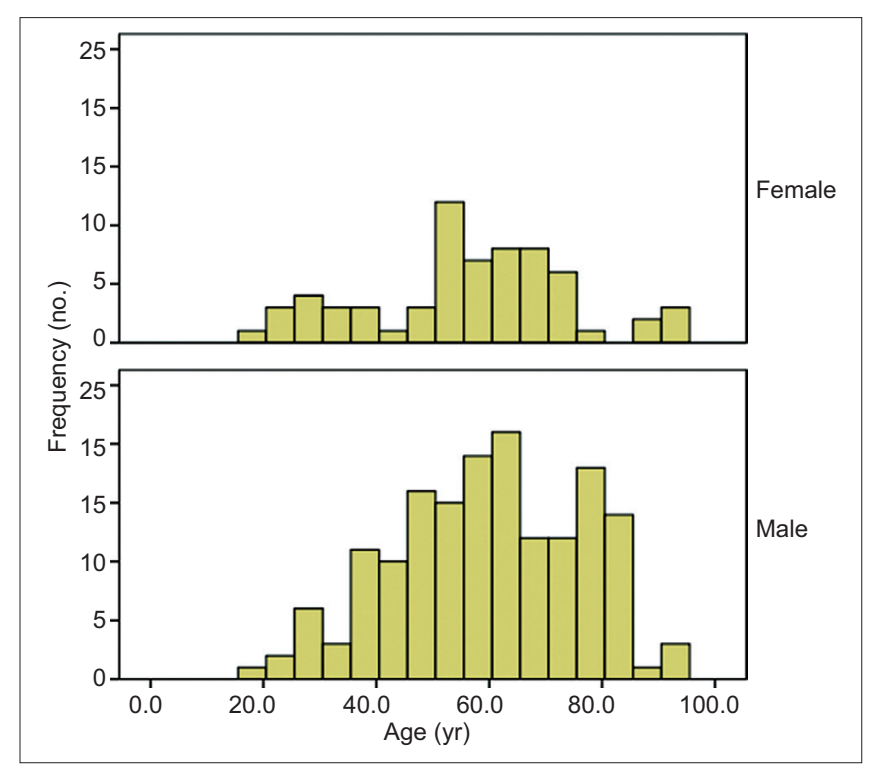

Fig. 1 Age distribution of patients by gender.

Table I. Characteristics of patients $(n=229)$.

\begin{tabular}{|ll|}
\hline Characteristic & No. (\%) \\
\hline Gender & \\
\hline Male & $164(71.6)$ \\
\hline Female & $65(28.4)$ \\
\hline Ethnicity & $160(69.9)$ \\
\hline Chinese & $18(7.9)$ \\
\hline Malay & $36(15.7)$ \\
\hline Indian & $15(6.6)$ \\
\hline Others
\end{tabular}

Data was retrieved as counts and percentages for the distributions of dermatophytes isolated, gender and ethnicity. Age distribution was depicted graphically via a histogram. A onesample chi-square test was used to compare the differences between the ethnic make-up of our patients and the general Singapore population. A p-value $<0.05$ was considered to be statistically significant.

\section{RESULTS}

There were 229 specimens that grew dermatophytes. Of these, $128(55.9 \%)$ specimens were from the dermatology outpatient clinic, $98(42.8 \%)$ were from inpatients seen at the hospital's Department of Dermatology and 3 (1.3\%) were from the day surgery centre. There were 164 (71.6\%) specimens isolated from men and $65(28.4 \%)$ specimens from women (male:female ratio of 2.5:1) (Table I \& Fig. 1). Ethnically, 160 (69.9\%) specimens were isolated from Chinese patients, 36 (15.7\%) from Indian patients, 18 (7.9\%) from Malay patients and $15(6.6 \%)$ from patients of other ethnicities (Table I). The ethnic make-up of our patients with tinea unguium caused by dermatophytes was different from that of the general Singapore population, which was $75.3 \%$ Chinese, $13.8 \%$ Malay, $8.6 \%$ Indian and $2.3 \%$ other ethnicities in 2006. ${ }^{(8)}$ In our cohort, patients of Indian and other ethnicities were over-represented, while Chinese and Malay patients were under-represented $(p=0.001)$. 
Table II. Patient distribution by age.

\begin{tabular}{|llll|}
\hline Age group $(\mathbf{y r})$ & \multicolumn{3}{c|}{ No. } \\
\cline { 2 - 4 } & Male & Female & Male + female \\
\hline $10-19$ & 1 & 0 & 1 \\
\hline $20-29$ & 6 & 6 & 12 \\
\hline $30-39$ & 14 & 7 & 21 \\
\hline $40-49$ & 26 & 7 & 33 \\
\hline $50-59$ & 33 & 17 & 50 \\
\hline $60-69$ & 35 & 15 & 50 \\
\hline $70-79$ & 29 & 8 & 37 \\
\hline $80-89$ & 17 & 1 & 18 \\
\hline $90-99$ & 3 & 4 & 7 \\
\hline Total & 164 & 65 & 229 \\
\hline
\end{tabular}

Table III. Dermatophytes isolated from nail specimen cultures ( $n=229$ ).

\begin{tabular}{|ll|}
\hline Dermatophyte & No. (\%) \\
\hline Trichophyton rubrum & $93(40.6)$ \\
\hline Trichophyton mentagrophytes & $60(26.2)$ \\
\hline Unidentified Trichophyton spp. & $57(24.9)$ \\
\hline Trichophyton tonsurans & $10(4.4)$ \\
\hline Epidermophyton floccosum & $5(2.2)$ \\
\hline Trichophyton verrucosum & $2(0.9)$ \\
\hline Trichophyton violaceum & $1(0.4)$ \\
\hline Trichophyton soudanense & $1(0.4)$ \\
\hline
\end{tabular}

The mean age of the patients was 58 (range 18-93) years. Although there is a neonatal department at $\mathrm{SGH}$, the tertiary hospital largely serves adult patients, and patients aged 50-59 years, 60-69 years and 70-79 years contributed the majority of our specimens ( $\mathrm{n}=137,59.8 \%$ ). Overall, patients aged over 50 years contributed 162 (70.7\%) specimens (Table II).

The most common dermatophytes isolated were Trichophyton rubrum $(40.6 \%)$, Trichophyton mentagrophytes $(26.2 \%)$ and unidentified Trichophyton spp. (24.9\%), followed by Trichophyton tonsurans, Epidermophyton floccosum, Trichophyton verrucosum, Trichophyton soudanense and Trichophyton violaceum (Table III).

\section{DISCUSSION}

Tinea unguium affects not only the cosmetic appearance of nails but also the patient's dexterity. It serves as a portal of entry for bacterial infection into the patient's skin and may even aid in spreading the dermatophyte infection to the skin and nails of other people in the community.

Nail clippings from the distal end of the nail are the usual specimens sent for culture to establish a diagnosis of onychomycosis before starting treatment. However, dermatophytes are often dead by the time they reach the distal end of the nail. This may possibly be because the dermatophyte is unable to survive in keratin, which is chemically much harder than the inner and middle nail plates that are its preferred habitat, or possibly due to loss of nutrients or moisture. ${ }^{(5)}$ Given this difficulty in successfully culturing dermatophytes from nail specimens, the likelihood of obtaining false-negative cultures remains high even for patients with nail infections. Diagnosing tinea unguium based primarily on laboratory cultures may thus suggest a false low prevalence that understates its actual magnitude in the general population.

Various methods have been adopted to overcome the difficulty in isolating fungi from nail clippings. One method is to use a dental drill fitted with a suction nozzle, as proposed by English. ${ }^{(5)}$ This instrument has raised the success rate of culture from microscopically positive nails from the usual rate of $50 \%$ to nearly $88 \% .^{(5)}$ However, the instrument is not practical for routine laboratory use.

To the best of our knowledge, there has been no study on the prevalence of tinea unguium in Singapore. In a prospective study done in the general adult population in Madrid, Spain, in 2000, the prevalence of tinea unguium was $2.8 \%$ (male $4.0 \%$, female $1.7 \%) .{ }^{(9)}$ This prevalence parallels that of psoriasis, ${ }^{(10)}$ which poses a significant health problem as well.

In our retrospective study over ten years, we found that most patients from whom dermatophytes were isolated were elderly $48.9 \%$ ( $n=112$ ) of dermatophyte specimens were from patients aged 60 years and above, and there were 37 (16.2\%) patients aged $70-79$ years and 25 (10.9\%) patients aged $80-99$ years. There are several reasons for tinea unguium being more common among elderly people. Young children seldom develop it because their nails grow faster than adults' nails, which reduces the chances of dermatophytes invading their nail plates. ${ }^{(6)}$ As people age, their nails grow at a slower rate, and thus there is a higher likelihood of their nail plates being invaded by dermatophytes. The nails of elderly people may also be subject to minor trauma due to poor eyesight, impaired mobility and poor nail care. Such dystrophic nails may be prone to invasion by dermatophytes, and even onycholysis and onychogryphosis. ${ }^{(6)}$ Other factors that predispose patients to tinea unguium include peripheral vascular diseases and diabetes mellitus, both of which are common comorbidities among elderly people.

In our study, more men than women were affected by tinea unguium (male:female ratio of 2.5:1). Our finding was similar to a study by Perea et al that found the male:female ratio among patients with tinea unguium in Spain to be 2.4:1. ${ }^{(9)}$ Men have tinea unguium more commonly than women because they tend to wear closed footwear and are more likely to have jobs and lifestyles that are predisposing factors.

Singapore is a multiethnic country with people of Chinese (majority), Malay, Indian and other ethnicities. In its general population, people of Indian and other ethnicities form a small proportion when compared to Chinese and Malay people. However, in our study, significantly higher proportions of patients of Indian and other ethnicities had tinea unguium when compared to Chinese and Malay patients. This was in contrast to a 1983 study by Ramesh et al, in which the incidence of toenail infection in India was found to be extremely low because the majority of people went barefoot. ${ }^{(1,11)}$

According to English, the most common dermatophytes that cause tinea unguium in Europe and North America were 
Trichophyton interdigitale (T. mentagrophytes var interdigitale) and T. rubrum. ${ }^{(5)}$ Similarly, Zaias also summarised in a review that the dermatophytes causing tinea unguium worldwide were T. rubrum and T. mentagrophytes in Europe and America. ${ }^{(12)}$ Hay listed E. floccosum, T. rubrum and T. interdigitale as the most common dermatophytes causing tinea unguium. ${ }^{(6)}$ In Hong Kong, Kam et al surveyed patients attending the Government Dermatology Clinics over an eight-year period from 1 January 1987 to 31 December 1994 and reported that, among 152 single infections with dermatophytes and yeasts, the most common dermatophytes were T. rubrum $(\mathrm{n}=68,44.7 \%)$, Trichophyton spp. $(\mathrm{n}=17,11.2 \%)$ and T. mentagrophytes $(\mathrm{n}=6,3.9 \%) .{ }^{(13)}$

$\mathrm{SGH}$ is a tertiary hospital with over 30 clinical departments that treat patients with multiple comorbidities, including solid organ transplant recipients, haematological patients, and oncology patients who are immunocompromised and more prone to infection, including fungal infection of the skin and skin appendages. Infections in immunocompromised patients may be caused by unusual pathogens, have atypical presentation, and run a protracted course with increased morbidity and mortality. In our study, the most common dermatophytes were T. rubrum (40.6\%), T. mentagrophytes (26.2\%) and unidentified Trichophyton spp. (24.9\%). Similar to Kam et al from Hong Kong, ${ }^{(13)}$ we found that T. rubrum was the most common cause of tinea unguium in Singapore and T. mentagrophytes was a common cause. Overall, T. rubrum was the most common dermatophyte that caused tinea unguium worldwide.(1) T. rubrum is an anthropophilic fungus that has become the most widely distributed dermatophyte in humans and frequently causes chronic infections of the skin and nails. ${ }^{(14)} T$. mentagrophytes was the second most common cause of tinea unguium in our study. We did not subspeciate T. mentagrophytes, however, as it is difficult to do so based merely on microscopic examination and phenotypic tests. For this reason, we were unable to establish how many of these specimen were T. interdigitale.

In the study by Hay, ${ }^{(6)}$ E. floccosum was one of the commonest dermatophytes causing tinea unguium. However, in our study, only $5(2.2 \%)$ specimens grew E. floccosum. Likewise, Kam et al also found that, among 152 single infections with dermatophytes and yeasts in Hong Kong, only $1(0.7 \%)$ specimen grew E. floccosum. ${ }^{(13)}$

There were a few uncommon species that caused tinea unguium in our study. T. tonsurans, a common cause of tinea capitis among Australian Aborigines and African Americans, ${ }^{(14)}$ was isolated from $10(4.4 \%)$ patients in our study. Although T. tonsurans can cause nail infection, it was not isolated in Hay's study. ${ }^{(6)}$ T. soudanense, an anthropophilic fungus that frequently causes tinea capitis in Africa, ${ }^{(14)}$ is rarely reported as a cause of tinea unguium. Its presence was not reported by Hay ${ }^{(6)}$ However, we had one patient with tinea unguium caused by T. soudanense. It is unlikely that this diagnosis was due to misidentification, as T. soudanense presents with a typical reflexive branching not seen in any other Trichophyton spp. on microscopic examination.

The natural habitat of $T$. verrucosum is cattle, in which it causes ringworm infection. It rarely causes infection among humans; when present, such infection is the result of close contact with cattle or infected fomites, causing very inflammatory tinea capitis, tinea barbae or tinea corporis. T. verrucosum has rarely been reported to cause nail infection. However, we reported two patients with tinea unguium caused by T. verrucosum. One of these patients was a renal transplant patient whose immunocompromised status may have predisposed her to the infection.

There were some limitations to our retrospective study. There were 57 specimens that grew Trichophyton spp. but were not further identified. Likewise, T. mentagrophytes was also not further subspeciated. Another limitation was that the cultured specimens were not categorised as fingernail or toenail infection, and that there was no correlation between the different dermatophytes and the various clinical features of tinea unguium.

In conclusion, our retrospective study at SGH over a ten-year period showed that $48.9 \%$ of patients with tinea unguium were aged 60 years and above. With the increasingly ageing population worldwide, tinea unguium should be expected to become an emerging health problem, as older people are more prone to the infection. We reported a higher proportion of patients of Indian and other ethnicities with tinea unguium in our cohort. T. rubrum was the most common dermatophyte isolated, while T. verrucosum, T. violaceum and T. soudanense were rare causes of tinea unguium in our study.

\section{ACKNOWLEDGEMENT}

The authors wish to acknowledge Mrs Tan Yang Ann MeeLee, from the Department of Microbiology, Singapore General Hospital, Singapore, for her help with retrieving the data from the department.

\section{REFERENCES}

1. Ramesh V, Reddy BS, Singh R. Onychomycosis. Int J Dermatol 1983; 22:148-52.

2. Hay RJ, Baran R. Fungal (Onychomycosis) and Other Infections Involving Nail Apparatus. In: Baran R, de Berker DA, Holberg M, Thomas L. Baran and Dawber's Diseases of the Nails and their Management. 4th ed. Oxford: WileyBlackwell, 2012.

3. Archen G, Wanet-Rouard J, Wiame L, van Hoff F. Les onychomycoses a moisissures: champigenons 'opportunistes'. Dermatologica 1979; 159:128-31.

4. Langer $\mathrm{H}$. [Epidemiology and clinical studies on onychomycosis; observations on Berlin's fungus flora, 1954-1956]. Arch Klin Exp Dermatol 1957; 204:62436. German.

5. English MP. Nails and fungi. Br J Dermatol 1976; 94:697-701.

6. Hay RJ. Infections affecting the nails. In: Samman PD, Fenton DA, eds. The Nails in Disease. 4th ed. London: William Heinemann, 1986.

7. Lim JT, Chua HC, Goh CL. Dermatophyte and non-dermatophyte onychomycosis in Singapore. Australas J Dermatol 1992; 33:159-63.

8. Department of Statistics, Ministry of Trade and Industry, Singapore. Population Trends 2016 [online]. Available at: https://www.singstat.gov.sg/-/media/files/ publications/population/population2016.pdf. Accessed August 15, 2018.

9. Perea S, Ramos MJ, Garau M, et al. Prevalence and risk factors of tinea unguium and tinea pedis in the general population in Spain. J Clin Microbiol 2000; 38:3226-30.

10. Van de Kerkhof P, Nestlé F. Psoriasis. In: Bolognia J, Schaffer J, Cerroni L. Dermatology. 4th Ed. Elsevier, 2018.

11. Desai SC, Bhatt ML. Dermatomycosis in Bombay: a study on incidence, clinical features, incriminating species of dermatophytes and their epidemicity. Indian J Med Res 1961; 49:662-71.

12. Zaias N. Onychomycosis: a review. Arch Dermatol 1972; 105:263-74.

13. Kam KM, Au WF, Wong PY, Cheung MM. Onychomycosis in Hong Kong. Int J Dermatol 1997; 36:757-61.

14. Ellis D, Kidd S. Trichophyton. In: Mycology Online. Available at: http://www. mycology.adelaide.edu.au/Fungal_Descriptions/Dermatophytes/Trichophyton/ rubrum.html. Accessed October 31, 2016. 